Original

\title{
Acoso sexual en el trabajo en la Administración Pública
}

\section{Sexual harassment at work in Civil Service}

\section{Fernando Mansilla Izquierdo}

Departamento de Salud Laboral. Madrid-Salud. Madrid. España.

Recibido: 07-02-14

Aceptado: 23-05-14

\section{Correspondencia}

Fernando Mansilla Izquierdo.

Departamento de Salud Laboral. Madrid-Salud. Madrid. España.

C/ Emilio Coll, n. 22, 1. 28224-Pozuelo de Alarcón. Madrid. España.

Correo electrónico: mansillaif@madrid.es

Resumen

Introducción: Se ha definido el acoso sexual como cualquier comportamiento sexual verbal, no verbal o físico no deseado que atente contra la dignidad de una persona y crea un entorno intimidatorio, humillante u ofensivo. Se trata de una conducta inesperada, inaceptable y ofensiva.

Objetivo: Explorar las características del acoso sexual en la administración pública y sus consecuencias.

Método: La muestra del presente estudio descriptivo ha estado formada por empleados públicos de las Administraciones: Local, Autonómica y Estatal. Se les aplicaron el Cuestionario Sociodemográfico y un Cuestionario de Acoso Sexual y a aquellos que puntuaron de forma positiva en el cuestionario de acoso sexual se realizó una entrevista y se les administraron un Cuestionario de Consecuencias del Acoso Sexual, el Inventario de Depresión Beck y el Cuestionario de Ansiedad Estado/Rasgo (STAI).

La aplicación de los instrumentos empleados se realizó de forma individual.

Resultados: Las características demográficas de la muestra que participó en el estudio dan como resultado que el perfil de la acosada sexualmente es una mujer de entre 20 y 40 años, soltera o separada, con formación de bachiller/FP o diplomatura y auxiliar administrativa.

No se han encontrado diferencias significativas en el porcentaje de acosados sexuales entre las distintas Administraciones Local, Autonómica y Estatal. El 2 \% de las trabajadoras fueron víctimas de acoso sexual.

Todas las trabajadoras que han sufrido acoso sexual han estado en tratamiento psicoterapéutico o farmacológico.

Manifiestan puntuaciones altas tanto en sintomatología ansiosa como en sintomatología depresiva.

Med Segur Trab (Internet) 2014; 60 (235) 335-343

Palabras clave: Acoso sexual. Administración pública. Trastorno de estrés postraumático.

Abstract

Introduction: Defined non-verbal or physical sexual harassment is any verbal sexual behavior, unwanted that violates the dignity of a person and creates an intimidating, humiliating or offensive environment. This is an unexpected, unacceptable and offensive behavior.

Objetive: Explore the characteristics of sexual harassment in the civil service and its consequences. 
Method: The sample of this research has been composed for Civil Service employees: Local, Regional and Central. The sample of this descriptive study was formed by employees of public authorities: Local, Regional and Central. They applied the Sociodemographic Questionnaire and Questionnaire Sexual Harassment and those who scored positively on the Questionnaire of Sexual Harassment an interview was conducted and were administered a Questionnaire Consequences of Sexual Harassment, the Beck Depression Inventory and the State/Trait Anxiety Inventory (STAI).

The application of the instruments used was performed individually.

Results: The demographic characteristics of the sample that participated in the study result that the profile of the abused is a woman between 20 and 40 years old, single or separate, with baccalaureate/FP or degree training and administrative assistant.

There were no significant differences in the percentage of sexual harassment between different Local, Regional and Central Administrations. 2\% of the workers were victims of sexual harassment.

All workers who have suffered sexual harassment have been in psychotherapy or drug treatment.

They show high scores on both anxiety symptoms and depressive symptoms.

Med Segur Trab (Internet) 2014; 60 (235) 335-343

Key words: Sexual Harassment. Civil Service. Post-Traumatic Stress Disorder. 


\section{INTRODUCCIÓN}

Aunque se ha señalado que en algunos casos de acoso laboral pueden darse también conductas que podrían estar dentro del ámbito del acoso sexual, es conveniente la distinción entre ambos. Esta diferencia estriba en que en el acoso sexual las conductas giran entorno al sexo, y en que la víctima de acoso sexual percibe las conductas de acoso de forma inmediata, mientras que las víctimas de acoso laboral tardan tiempo en percibir las conductas de acoso. Ambos tienen características comunes como la situación de humillación y de ataque a la dignidad que sufren las personas en ambas situaciones, pero el acoso sexual tiene especifidad por el objetivo de la conducta del acosador y por el tipo de conductas. También se ha señalado que el acosador laboral a diferencia del sexual mantiene siempre la convicción interna de no haber hecho nada malo, aún después de la condena, sin embargo el acosador sexual termina por reconocer que ha realizado alguna conducta inapropiada ${ }^{(1)}$. Y las víctimas de acoso laboral suelen sufrir más consecuencias adversas que las de acoso sexual ${ }^{(2)}$.

En el fenómeno del acoso sexual hay que tener en cuenta que no se trata de

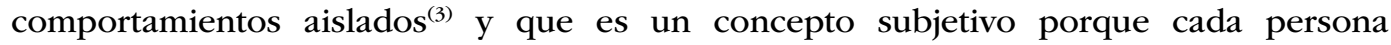
determina el comportamiento que aprueba o tolera, ya que los que son más tolerantes con las conductas de acoso sexual perciben menos las conductas de acoso sexual ${ }^{(4)}$.

El acoso sexual en las organizaciones se ve favorecido por aspectos organizativos como la sexualización del entorno de trabajo, la proporción de hombres-mujeres, el tipo de tareas que realizan, la discriminación sexual, el clima laboral o la valoración del trabajo $^{(5)}$.

El acoso sexual es un fenómeno social de múltiples y diferentes dimensiones, denunciado por distintas organizaciones e instituciones y constatado por distintas investigaciones que han evidenciado la existencia, extensión y gravedad en el ambiente laboral.

Según la OIT $^{(6,7)}$, para que haya acoso sexual deben integrarse tres elementos: un comportamiento de carácter sexual, que no sea deseado y que la víctima lo perciba como un condicionante hostil para su trabajo. El acoso sexual es cualquier tipo de acercamiento o presión de naturaleza sexual tanto física como verbal, no deseada por quien la sufre, que surge de la relación de empleo y que da por resultado un ambiente de trabajo hostil, un impedimento para hacer las tareas y un condicionamiento de las oportunidades de ocupación de la persona perseguida.

En el Código Penal Español se define al acosador como el que solicita favores de naturaleza sexual, para sí o para un tercero, en el ámbito de una relación laboral, docente o de prestación de servicios, continuada o habitual, y con tal comportamiento provoca a la víctima una situación objetiva y gravemente intimidatoria, hostil o humillante.

En la recomendación de la Comunidad Europea de 27 de noviembre de 1991, relativa a la dignidad de la mujer y del hombre en el trabajo se define el acoso sexual como la conducta de naturaleza sexual u otros comportamientos basados en el sexo que resulta inaceptable, indeseada, irrazonable y ofensiva para la persona que es objeto de la misma.

La Directiva 2002/73/CE del Parlamento Europeo y del Consejo, de 23 de septiembre de 2002, define el acoso sexual como la situación en que se produce cualquier comportamiento verbal, no verbal o físico no deseado de índole sexual con el propósito o el efecto de atentar contra la dignidad de una persona, en particular cuando se crea un entorno intimidatorio, hostil, degradante, humillante u ofensivo.

La conducta verbal de naturaleza sexual puede incluir insinuaciones sexuales molestas o comentarios obscenos, proposiciones o presión para la actividad sexual; insistencia para una actividad social fuera del lugar de trabajo después que se haya puesto en claro que dicha insistencia es molesta. 
En la conducta no verbal de naturaleza sexual quedarían incluidas la exhibición de fotos sexualmente sugestivas o pornográficas, de objetos o materiales escritos, las miradas impúdicas, los silbidos o hacer ciertos gestos.

A cada persona le corresponde determinar el comportamiento que aprueba o tolera, lo que imposibilita el hacer una relación de conductas vejatorias. Por tanto, la determinación de qué comportamientos resultan o no molestos es algo que depende del receptor de las conductas, siendo en este punto irrelevante la intencionalidad del emisor de las conductas $^{(8)}$.

Por tanto, el acoso sexual consiste en la acción impuesta sin reciprocidad, inesperada y no bien recibida, frecuente y repetitiva que puede tener un efecto devastador en la víctima. Puede incluir tocamientos, insinuaciones, miradas, actitudes chocantes, bromas con lenguaje ofensivo, alusiones a la vida privada, referencias a la orientación sexual, insinuaciones con connotación sexual, alusiones a la figura y a la ropa, etc.

\section{OBJETIVO}

Explorar las características del acoso sexual en la administración pública y sus consecuencias, y valorar la necesidad de recomendar medidas preventivas.

\section{MÉTODO}

\section{Participantes}

La muestra de este estudio transversal y descriptivo ha estado formada por empleados públicos de las tres Administraciones (N: 300) Local (n: 100), Autonómica (n: 100) y Estatal (n: 100) seleccionados de forma aleatoria. De los 300 sujetos de la muestra 150 eran varones y 150 mujeres. A pesar de que en las distintas Administraciones las mujeres son el $52,71 \%$ de la plantilla y los hombres el 47,29\% (Datos del Ministerio de la Presidencia. Secretaría del Estado para la Función Pública), se estableció una muestra con igual número de hombres y de mujeres en las distintas Administraciones: en la Administración Local (50 hombres y 50 mujeres), en la Autonómica (50 hombres y 50 mujeres) y en la Estatal (50 hombres y 50 mujeres) (Tabla I). Todos los sujetos eran administrativos o auxiliares administrativos y edades comprendidas entre los 20 y los 60 años.

Tabla I. Muestra

\begin{tabular}{lcccc}
\hline & LOCAL & AUTONÓMICA & ESTATAL & TOTAL \\
\hline HOMBRES & 50 & 50 & 50 & 150 \\
\hline MUJERES & 50 & 50 & 50 & 150 \\
\hline TOTAL & 100 & 100 & 100 & 300 \\
\hline
\end{tabular}

\section{Procedimiento}

A los sujetos que participaron se les informó del objetivo y las características del estudio. La aplicación de los instrumentos empleados se realizó de forma individual y se llevó a cabo durante el segundo semestre de 2013.

En una primera fase se les administró el Cuestionario Sociodemográfico y el Cuestionario de Acoso Sexual; a aquellos que dieron una puntuación positiva en el Cuestionario de Acoso Sexual, se les entrevistó y se les aplicó el Inventario de Depresión Beck y el Cuestionario de Ansiedad Estado/Rasgo (STAI). Se les solicitó que respondiesen a estos cuestionarios con los sentimientos de cuando ocurrió la situación de acoso. 


\section{Instrumentos}

Se administraron un Cuestionario Sociodemográfico y un Cuestionario de Acoso Sexual (consta de 12 ítems y evalúa la percepción subjetiva de ser objeto de acoso sexual). A aquellos que puntuaron de forma positiva en el Cuestionario de Acoso Sexual, se les realizó una entrevista y se les administraron un Cuestionario de Consecuencias del Acoso Sexual, el Inventario de Depresión Beck (desarrollado, en un primer momento, como un instrumento heteroaplicado de 21 ítems que evaluaba la intensidad del síndrome depresivo) y el Cuestionario de Ansiedad Estado/Rasgo (STAI) El Cuestionario STAI evalúa dos conceptos: Ansiedad como estado (A/E) sobre el estado emocional transitorio, caracterizado por sentimientos subjetivos, conscientemente percibidos, de atención y aprensión y por hiperactividad del sistema nervioso autónomo. Ansiedad como rasgo (A/R) señala una propensión ansiosa, relativamente estable, que caracteriza a los individuos con tendencia a percibir las situaciones como amenazadoras.

\section{RESULTADOS}

Los datos fueron analizados con programa estadístico informático Statistical Package for the Social Sciences (SPSS 16). Se realizaron análisis descriptivos de las diferentes variables sociodemográficas (Tabla II), así como los datos clínicos (Tabla III).

Tabla II. Datos sociodemográficos de las víctimas de acoso sexual

\begin{tabular}{|c|c|c|c|c|c|c|c|c|}
\hline & \multicolumn{2}{|c|}{ LOCAL } & \multicolumn{2}{|c|}{ AUTONÓMICA } & \multicolumn{2}{|c|}{ ESTATAL } & \multicolumn{2}{|c|}{ TOTAL } \\
\hline & n & $\%$ & $\mathbf{n}$ & $\%$ & n & $\%$ & n & $\%$ \\
\hline $\mathrm{N}=6$ & 1 & $17 \%$ & 3 & $50 \%$ & 2 & $33 \%$ & 6 & $100 \%$ \\
\hline \multicolumn{9}{|l|}{ GÉNERO } \\
\hline Hombre & 0 & & 0 & & 0 & & 0 & \\
\hline Mujer & 1 & $17 \%$ & 3 & $50 \%$ & 2 & $33 \%$ & 6 & $100 \%$ \\
\hline \multicolumn{9}{|l|}{ EDAD } \\
\hline $25-30$ & 1 & $17 \%$ & 1 & $17 \%$ & & & 2 & $33 \%$ \\
\hline $31-40$ & & & 2 & $33 \%$ & 1 & $17 \%$ & 3 & $50 \%$ \\
\hline $41-50$ & & & & & & & 0 & \\
\hline $51-65$ & & & & & & & 0 & \\
\hline \multicolumn{9}{|l|}{ ESTADO CIVIL } \\
\hline Soltero/a & 1 & $17 \%$ & 1 & $17 \%$ & & & 2 & $33 \%$ \\
\hline Casado/a-En pareja & & & & & & & 0 & \\
\hline Separado/a-divorciado/a & & & 2 & $33 \%$ & 1 & $17 \%$ & 3 & $50 \%$ \\
\hline Viudo/a & & & & & & & 0 & \\
\hline \multicolumn{9}{|l|}{ NIVEL DE ESTUDIOS } \\
\hline Licenciado & & & & & 1 & $17 \%$ & 1 & $17 \%$ \\
\hline Diplomado & & & 2 & $33 \%$ & & & 2 & $33 \%$ \\
\hline Bachiller/Formación Prof. & 1 & $17 \%$ & 1 & $17 \%$ & & & 2 & $33 \%$ \\
\hline EGB/Estudios Primarios & & & & & & & 0 & \\
\hline \multicolumn{9}{|l|}{ PUESTO DE TRABAJO } \\
\hline Administrativo & & & & & & & 0 & \\
\hline Auxiliar administrativo & 1 & $17 \%$ & 3 & $50 \%$ & 2 & $33 \%$ & 6 & $100 \%$ \\
\hline \multicolumn{9}{|l|}{ TIPO DE CONTRATO } \\
\hline Funcionario & & & & & & & 0 & \\
\hline Funcionario interino & 1 & $17 \%$ & 1 & $17 \%$ & 1 & $17 \%$ & 3 & $50 \%$ \\
\hline Laboral & & & 2 & $33 \%$ & & & 2 & $33 \%$ \\
\hline Laboral eventual & & & & & & & 0 & \\
\hline
\end{tabular}


Tabla III. Datos clínicos de las víctimas de acoso sexual

\begin{tabular}{ll}
\hline$\overline{\mathrm{X}}$ de tratamiento en salud mental & 9 meses \\
\hline$\overline{\mathrm{X}}$ de tiempo en Incapacidad Laboral Transitoria & 4 meses \\
\hline$\overline{\mathrm{X}}$ del resultado en el Inventario Beck & depresión moderada-grave \\
\hline$\overline{\mathrm{X}}$ de puntuación Cuestionario STAI & centil 81 \\
\hline
\end{tabular}

No hubo casos de acoso sexual de hombres en ninguna de las tres Administraciones, pero hubo 1 caso de acoso sexual en la Administración Local, 3 casos en la Administración Autonómica y 2 casos en la Administración Estatal.

El perfil de la acosada sexualmente es una mujer de entre 20 y 40 años, soltera o separada ( 2 eran solteras y 4 separadas o divorciadas), funcionaria interina o contratada eventual ( 5 eran funcionarias interinas y 1 contratada laboral), con formación de bachiller/ FP o titulado grado medio o titulado grado superior ( 2 eran bachiller/FP, 3 diplomadas y 1 licenciada), con puesto de trabajo de auxiliar administrativa (las 6 eran auxiliares administrativo).

Además, todas las trabajadoras que han sufrido acoso sexual han estado en tratamiento psicoterapéutico o farmacológico durante un periodo de 7 a 13 meses y han permanecido en Incapacidad Laboral Transitoria (ILT) de 3 a 6 meses (con una media de 4 meses de duración).

Las trabajadoras que dieron un resultado positivo en el Cuestionario de Acoso Sexual, también puntuaron positivamente en el Inventario de Depresión de Beck, por encima de 21 (depresión moderada-grave) y en el Cuestionario de Ansiedad STAI-Estado (entre el centil 70 y el 99). Por tanto, manifestaron puntuaciones altas tanto en sintomatología ansiosa como depresiva. La totalidad de las que dieron un resultado positivo en acoso sexual no presentaron alteraciones de memoria, ni sensoperceptivas, ni del curso y contenido del pensamiento, pero si manifestaron un estado depresivo-ansioso con tristeza, retraimiento social, nerviosismo, tensión, insomnio, llanto, cansancio, pérdida de apetito y peso, actitud de desánimo, irritabilidad, sentimiento de culpabilidad, disminución de la efectividad en el trabajo y pérdida de interés por el sexo, sintomatología compatible con el trastorno por estrés postraumático (CIE: F43.1).

\section{DISCUSIÓN Y CONCLUSIONES}

Este trabajo confirma como otros estudios que la sintomatología que refieren las víctimas de acoso pueden ser concomitante con el trastorno de estrés postraumático $(\mathrm{TEP})^{(9,10)}$.

En nuestro estudio no se han encontrado diferencias significativas en el porcentaje de acosados sexuales entre las distintas Administraciones Local, Autonómica y Estatal. El porcentaje del $2 \%$ de la muestra de trabajadoras, es menor que en algunos otros estudios como el desarrollado por la III Encuesta Europea sobre Condiciones de Trabajo del año 2000, que indicó que un 3\% de mujeres fueron víctimas de acoso sexual en el año anterior a la realización de dicha encuesta. También en España, la encuesta de la Secretaría de la Mujer de Comisiones Obreras (año 2000), señala que un 14,5\% de los trabajadores ha vivido una situación de acoso sexual a lo largo de su vida laboral. En la IV Encuesta Europea sobre Condiciones de Trabajo del año 2007 se afirma que el acoso sexual afecta al $10 \%$ de las trabajadoras checas y al $7 \%$ de las noruegas y al $6 \%$ de las croatas y turcas, mientras que las trabajadoras italianas y españolas tienen un índice de menos del $1 \%$. Aunque surge una discrepancia estadística entre estudios, ya que ese mismo año en un estudio realizado por el Instituto de la Mujer en el 2007 se subraya que el 14,9\% de las mujeres trabajadoras en España ha sufrido alguna situación de acoso sexual en el último año. Por otro lado en nuestro trabajo se indica que las mujeres que sufrieron acoso 
manifestaron sintomatología ansioso-depresiva ${ }^{(11)}$ y confirma que las mujeres sufren la conducta de acoso más que los hombres, quizá porque los hombres y mujeres difieren en la conciencia de qué constituye acoso sexual ${ }^{(12)}$. Pero la IV Encuesta Europea sobre Condiciones de Trabajo del año 2007 corrobora que el acoso sexual afecta tres veces más a las trabajadoras que a los trabajadores, ya que de nuestra muestra de 150 hombres y 150 mujeres ningún hombre dijo haber sido acosado sexualmente a lo largo de su vida laboral y sí lo afirmaron 6 mujeres. Aunque se ha apuntado que las trabajadoras sean acosadas sexualmente tres veces más que los trabajadores, puede deberse a los ámbitos de ocupación de las mujeres, la segregación de las mujeres a los trabajos peor pagados y de menor categoría y quizá la subordinación jerárquica e inestabilidad en el empleo de la mujer ${ }^{(13)}$.

Aunque se ha afirmado que el $88 \%$ de las mujeres universitarias declaraban haber presenciado acoso sexual, y sólo el 5,6\% admitía haberlo sufrido ${ }^{(14)}$, en nuestro estudio se muestra que en la Administración Pública son las administrativas y operarias las personas objeto del acoso sexual, lo que confirma que la creencia generalizada de que el acoso sexual está relacionado con los cánones de belleza, es un mito, ya que el acoso sexual tiene más bien que ver con las relaciones de poder ${ }^{(15)}$ y con la jerarquía, que añade una carga de desamparo, según se pudo corroborar en nuestro estudio, ya que en todos los casos el acosador era su jefe. Y también confirma que el acoso sexual podría venir potenciado por una situación laboral precaria ${ }^{(16)} \mathrm{o}$ más concretamente al empleo, a la continuidad del contrato de trabajo, etc. ${ }^{(17)}$, ya que todas las trabajadoras acosadas sexualmente eran interinas.

En nuestro estudio las trabajadoras no habían sufrido acoso sexual durante el último año, pero sí a lo largo de su experiencia laboral.

En nuestro estudio la edad media del grupo de mayor riesgo de sufrir acoso sexual es menor a 30 años, y no coincide con los resultados obtenidos en la III Encuesta Europea sobre Condiciones de Trabajo del año 2000 llevada a cabo por la Fundación Europea para la Mejora de las Condiciones de Vida y de Trabajo, que señala que el grupo de más riesgo son mujeres menores de 30 años. Y coincide más con el estudio en el que las mujeres afectadas son de menos de 34 años y solteras, pero no coincide con la OIT que ve el perfil más probable de la víctima como una mujer viuda, separada o divorciada, con contrato irregular o recién ingresada en el mercado laboral y que trabaja en entorno masculino.

Se ha afirmado en el estudio sobre el acoso sexual en España publicado por Comisiones Obreras en noviembre de 2000 que el acoso sexual se puede producir entre personas de todo el escalafón laboral, tanto entre profesionales como entre los trabajadores de menor cualificación y que el acoso sexual no tiene edad, que puede afectar por igual a una joven de veinte años que a una trabajadora de cuarenta y que la víctima suele estar separada o divorciada y en situación de precariedad laboral. Nuestro estudio en la Administración no confirma estos datos, ya que el perfil de la acosada sexualmente es una mujer de entre 20 y 40 años, soltera o separada funcionaria interina o contratada eventual, con formación de bachiller/FP o titulado grado medio o titulado grado superior, con puesto de trabajo de auxiliar administrativa y con experiencia laboral de entre 4 y 7 años.

Para concluir, queremos resaltar la necesidad de que las empresas más afectadas por este riesgo establezcan un Políticas y Planes de Prevención con el desarrollo de medidas preventivas efectivas encaminadas a disminuir el riesgo de acoso sexual en el trabajo.

- Es sabido que el impacto del acoso sexual está moderado por la vulnerabilidad de la persona, y afecta negativamente tanto al trabajador ${ }^{(18)}$ como al proceso productivo, ya que genera absentismo, bajas por enfermedad, menor productividad debido al descenso de la cantidad y calidad del trabajo y a la menor motivación para el trabajo. $Y$ aunque las consecuencias del acoso sexual afectan fundamentalmente a la persona contra la cual se ejerce el acoso, también incide negativamente sobre los trabajadores que pueden ser testigos o conocer el problema. Por lo tanto, es necesario y preventivo que las empresas y la 
administraciones establezcan Políticas Preventivas contra el acoso sexual que contemplen el Art. 62 de la Ley Orgánica 3/2007, de 22 de marzo, para la igualdad efectiva de mujeres y hombres, en el que se establece la realización de un protocolo de actuación frente al acoso sexual y al acoso por razón de sexo, porque se ha encontrado una relación entre los aspectos específicos de género de clima organizacional y el grado de comportamiento sexual no deseado ${ }^{(19)}$.

- La aprobación en Comité de Seguridad y Salud (CSS) de un protocolo para la prevención, detección y actuación contra las situaciones de acoso sexual en las empresas, así como sus recomendaciones y procedimientos de actuación derivados, constituyen una fuente de prevención de conductas no deseadas. Ya que se ha demostrado que las características del centro de trabajo, incluyendo la gestión y estrategias, están relacionadas con el riesgo de acoso sexual ${ }^{(20)}$, incluso que los factores ambientales de la organización predicen el acoso sexual ${ }^{(21)}$.

Por todo ello, la organización de la empresa debe asegurarse la adecuada difusión de la política contra el acoso, de manera que sea comunicada a todos los trabajadores y de que estos sepan que tienen un derecho de queja y de que existe un firme compromiso en no tolerar los comportamientos de acoso sexual (información a los trabajadores). Además la responsabilidad de asegurar un entorno de trabajo respetuoso es de todos los trabajadores, recomendándose a los mandos que tomen medidas para promocionar la política de no acoso sexual (formación en prevención del acoso en el lugar de trabajo).

\section{BIBLIOGRAFÍA}

1. Gimeno Lahoz, R (2004). La Presión Laboral Tendenciosa (Mobbing). Tesis Doctoral. Universidad de Girona.

2. Hershcovics, MS, Barling, J. (2010). Comparing victim attributions and outcomes for workplace aggression and sexual harassment. Journal of Applied Psychology, 95, 874-888.

3. Pernas, B, Roman, M, Olza, J, Naredo, M (2000). La dignidad quebradiza: Las raíces del acoso sexual en el trabajo. Madrid: La Catarata.

4. McCabe, MP, Hardman, L (2005). Attitudes and perceptions of workers to sexual harassment. Journal of Social Psychology, 145, 6, 19-40.

5. Llaneza Álvarez, FJ (2002). Ergonomía y Psicosociología Aplicada. Manual para la formación del especialista. Valladolid: Editorial Lex Nova.

6. OIT (1995). La lucha contra el acoso sexual. Madrid: INSHT.

7. OIT (1997). Desagradable, no deseado y cada vez más ilegal: el acoso sexual en el lugar de trabajo. Revista Trabajo, 19, 24-29.

8. Penix, T, O’Donohue, W (2000). Sexual Harassment. Annual Review of Sex Research, 11, $258-285$.

9. Echeburúa, E, De Corral, P, Zubizarreta, I, Sarausa, B (1995). Trastorno de estrés postraumático crónico en víctimas de agresiones sexuales. A Coruña. Fundación Paideia.

10. Larsen, SE, Fitzgerald, LF (2011). PTSD symptoms and sexual harassment: the role of attributions and perceived control. Journal of Interpersonal Violence, 26,13, 2555-2567.

11. Valente, SM, Bullough, V (2004). Sexual harassment of nurses in the workplace. The Journal of Nursing Care Quality, 19, 3, 234-241.

12. Banerjee, A, Sharma, B (2011). Gender differences in perception of workplace sexual harassment among future professionals, Industrial Psychiatry Journal, 20, 21-24.

13. Rotundo, M, Nguyen, DH, Sackett, PR (2001). A Meta-Analytic review of gender differences in perceptions of sexual harasssment. Journal of Applied Psychology, 86, 5, 914-922.

14. Brooks, L, Perot, AR (1991). Reporting sexual harassment: Exploring a predictive model. Psychology of Women Quaterly, 15, 31-47.

15. INSHT (2001). Mobbing, violencia física y acoso sexual. Madrid: Ministerio de Asuntos Sociales.

16. INSHT (1999). NTP 507: Acoso sexual en el trabajo. Madrid: INSHT. Ministerio de Trabajo y Asuntos Sociales. 
17. González de Rivera, JL (2002). El maltrato psicológico: cómo defenderse del mobbing y otras formas de acoso. Madrid: Espasa Calpe.

18. Nielsen, MB, Eionarsen, S (2012). Prospective relationships between workplace sexual harassment and psychological distress. Occupational Medicines, 62, 3, 226-228.

19. Timmerman, G, Bajema, C (2000). The impact of organizacional culture on perceptions and experiences of sexual harassment. Journal of Vocational Behavior, 57, 2, 188-205.

20. Zinder JA, Scherer, HL, Fisher, BS (2012). Social organization and social ties: their effects on sexual harassment victimization in the workplace. Work, 42, 1, 137-150.

21. Topa Cantisano, G, Morales Domínguez, JF, Depolo, M (2008). Perceived sexual harassment at work: metaanalysis and structural model of antecedents and consequences. The Spanish Journal of Psychology, 11, 1, 207-218.

|| 